\title{
PRESENTACIÓN
}

\section{WEB 2.0: DISPOSITIVOS MÓVILES Y ABIERTOS PARA EL APRENDIZAJE"}

\author{
Dr. Alejandro Pisanty (coordinador) \\ México D.F.
}

Este número de la Revista Iberoamericana de Educación a Distancia (RIED) es producto de una invitación del estimado Profesor Lorenzo García Aretio al autor de esta nota, para reunir en un número especial algunos artículos enfocados a aspectos tecnológicos de la educación a distancia. Conté para esta tarea, y lo agradezco profundamente, con el apoyo generoso de Larisa Enríquez Vázquez y de Juan Cristóbal Cobo Romaní. Por parte de la RIED, Lorenzo García Aretio, Marta Ruiz Corbella y María García Pérez hicieron contribuciones invaluables con su estímulo, visión, orden, rigor, y paciencia. Por esta última en especial tengo un agradecimiento sin límites; por las otras virtudes, profundo reconocimiento.

La tecnología que se utiliza en educación a distancia, o mejor dicho en plural, las tecnologías, se encuentran en constante evolución. Tanto entran en uso en educación a distancia tecnologías que se desarrollan para otros fines cuanto se producen en educación a distancia innovaciones que se propagan mucho más allá de las fronteras del campo. Las tecnologías dan forma a las actividades, bien por facilitarlas o bien por limitarlas, y por otra parte las actividades y las comunidades fuerzan la creación de nueva tecnología y acompañando a ésta nuevos paradigmas.

En los últimos años, los cambios mundiales en conceptos tan alejados aparentemente de la tecnología como la propiedad intelectual - en modalidades como "Creative Commons" o Acceso Abierto, así como la difusión intensiva de las telecomunicaciones móviles, sumados a la constante construcción, expansión y consolidación de la educación a distancia han traído consigo la existencia de gradaciones sutiles entre muchos modelos posibles y que hace una década estaban totalmente diferenciados. El aprendizaje en dispositivos móviles cuenta con el respaldo de acervos y sistemas altamente adaptables y accesibles en línea, los modelos "mixtos" o "blended" que varían entre aprendizaje presencial y situaciones sin contacto físico alguno, en fin, la variación lleva a una adaptabilidad de grano muy fino y evolución constante. 
Para el número que ahora presentamos, los resultados de nuestra convocatoria dieron como resultado un conjunto de valiosos artículos. Quizás sorprendentemente, todos menos uno hacen recuento de experiencias de aprendizaje móvil. Todos están fundados en sólidas bases teóricas y buenos trabajos empíricos. A algunos lectores les sorprenderá que no haya una colección de trabajos basados en el estudio de las "redes sociales" (si bien el de Pardo Kuklinski toca el tema) u otros temas que también son de vivo interés en la actualidad. Nuestra observación es que mucho de ese trabajo (por ejemplo el muy notable que lleva a cabo Alejandro Piscitelli) está en proceso y los artículos de la profundidad requerida por la RIED apenas están siendo producidos. Otras tecnologías, como las aplicaciones de la inteligencia artificial, los buscadores de gran escala y su personalización, y otras están en un hiato entre ciclos, y la gran producción de la nueva ola está por empezar. Y finalmente, hay un limite a lo que la RIED, y este autor, pueden convocar en un plazo finito y para una revista de tamaño no menos finito.

Hugo Pardo Kuklinski presenta una aplicación práctica de los conceptos de Web 2.0 sobre dispositivos móviles que ha construido en sus importantes aportaciones a la literatura del campo. Propone y presenta un sistema, ya en operación, que permite potenciar enormemente el uso de los dispositivos móviles de telecomunicaciones para la educación.

El artículo de Hugo Pardo Kuklinski nos llega desde la frontera, el "borde cortante", de los desarrollos de la tecnología y sus modelos de utilización. Pardo Kuklinski expone las condiciones del mercado de comunicaciones móviles, particularizando para el caso de España pero en términos que se aplican más ampliamente, y apunta en particular a las relaciones de poder que este mercado manifiesta entre proveedores y usuarios.

Pardo Kuklinski extiende su análisis como un marco que permite comprender el elevado valor de su iniciativa "campusmovil.net" en términos de una subversión necesaria de esas relaciones de poder. Al centrar las actividades continuas y cotidianas en el dispositivo móvil, y al dar al usuario una identidad basada en Internet en lugar de una basada en el número de teléfono, lo libera de algunos de los lazos más fuertes a que lo somete el proveedor.

Este avance puede ser entendido como la aplicación exitosa de décadas de construcción de la filosofía de Internet, basada en principios como la abstracción del funcionamiento de la red en un modelo de capas, el principio "end-to-end" o de punta a punta, y el principio de "red tonta - inteligencia en la orilla", que 
deposita la capacidad de las aplicaciones y la riqueza del software y la información en los dispositivos más cercanos al usuario, y no en el corazón de la red donde las monetizaría y dominaría el operador de la red.

Es notable también en el artículo de Pardo Kuklinski que no muestre inhibiciones al hablar de la monetización de las ideas expuestas y en cambio discuta abiertamente los antecedentes y restricciones a que se enfrenta dicha monetización. Si bien este grado de liberalismo acalambra a muchos miembros de las comunidades académicas de habla hispana, especialmente en las instituciones públicas, es extremadamente sano ponerlo sobre la mesa (así sea ésta la de disecciones) para con franqueza analizar la viabilidad y sustentabilidad de los valiosos servicios y avances que impulsan Pardo Kuklinski y las personas y grupos que se asocian con ellos.

El trabajo encabezado a lo largo de ya varios años por Alfonso Bustos dedica cuidadosa atención a la escritura colaborativa en línea. En el artículo que se integra al presente volumen, empieza por una discusión que merece amplia lectura y se refiere al papel que juega la escritura en general, y en particular en educación. Desde una visión filosófica del tema, pasa a instancias específicas y finalmente a un trabajo muy puntual con un grupo de alumnos.

Algunas de las instancias de la escritura estudiadas en este trabajo enfocan la atención en los valores educativos que aporta la escritura en línea, así como las facilidades que ofrece a los educadores para comprender a fondo los procesos de la escritura entre los estudiantes y las formas en que es aprovechada para construir aprendizajes. El seguimiento de actividades detallado que es posible realizar con algunas herramientas informáticas puede ayudar a producir conocimiento si se recogen e interrogan debidamente datos relevantes.

El grupo de estudio, subdividido a su vez para los fines del experimento conducido, realizó tareas de escritura colaborativa en tres formas diferentes, una síncrona mediante un "chat", un foro en línea asíncrono, y un medio colaborativo en un Wiki. Los resultados ilustran conductas de los alumnos que resultan fácilmente discernibles en las bitácoras de los sistemas, y permiten identificar los casos en que la colaboración pasa de la suma de aportaciones para que un solo miembro de un equipo escriba, a una escritura colaborativa, y a una escritura en la que el carácter colaborativo es tan intenso que se establece una verdadera coautoría entre los participantes. 
El trabajo, que debe mucho tanto a los trabajos empíricos y primeras aproximaciones conceptuales alcanzadas en la UNAM como posteriormente a la colaboración en Barcelona con Coll y otros investigadores, ofrece entonces una lectura rica en conceptos y un conjunto de bases para interrogar a la realidad que dará fruto en estudios posteriores.

Ma. Soledad Ramírez Montoya presenta las investigaciones sobre aprendizaje móvil o m-learning que se llevan a cabo en el Instituto Tecnológico de Estudios Superiores de Monterrey (ITESM). Presenta primeramente una visión de conjunto del m-learning, partiendo de su evolución y el debate que ocurre alrededor de la tarea de definirlo.

De allí, Ramírez Montoya hace una revisión del estado del m-learning en el ITESM, mostrando diversos desarrollos y usos entre alumnos y académicos de esa institución. Finalmente, presenta un amplio e interesante conjunto de investigaciones que la institución está pudiendo realizar con base en las actividades de aprendizaje móvil que se llevan a cabo en ella.

Este conjunto de investigaciones abarca una variedad de campos, como por ejemplo las variables que determinan la mayor o menor utilización de recursos de m-learning por parte de alumnos de las generaciones más jóvenes, las interacciones educativas y sociales necesarias para que el m-learning produzca mejores resultados, o las competencias tecnológicas y otros conocimientos necesarios para la producción de recursos educativos de aprendizaje móvil.

Finalmente, Ramírez Montoya hace una revisión desinhibida de los factores adversos que el m-learning encuentra en la actualidad, debido a las limitaciones de los dispositivos en uso. Entre éstas encuentra complicaciones debidas a las limitaciones físicas de los dispositivos (despliegue, entrada de información, memoria) y las derivadas de la operación de las redes inalámbricas, específicamente las de telecomunicaciones móviles comerciales, que por su alto costo y operación deficiente pueden dificultar enormemente los proyectos. En complemento, expone algunas líneas de trabajo a futuro que sin duda deberán ser seguidas por diversos investigadores.

Fernando Mortera expone el diseño, construcción y puesta en marcha del proyecto KnowledgeHub en el mismo ITESM. Este proyecto es un portal centralizado de acceso a publicaciones de acceso abierto para la comunidad académica del ITESM. 
KnowledgeHub es un portal desarrollado con Drupal que da acceso diferenciado a los recursos que cataloga, de tal manera que permite diversos permisos para editar o solamente acceder para la lectura a los documentos.

Uno de los impactos a largo plazo que sería deseable tenga este proyecto es que la comunidad del ITESM adquiera un gran aprecio por el valor de compartir los productos del trabajo del conocimiento que hacen quienes los aportan al público bajo la filosofía de acceso abierto, y hagan lo propio con los suyos al desarrollarse profesionalmente afuera de la institución o académicamente dentro de ella.

El número aquí presentado reúne, entonces, contribuciones diversas y enfoques complementarios. De su lectura y estudio esperamos que los lectores puedan derivar aplicaciones inmediatas y reflexiones para continuar con la expansión del conocimiento sobre la educación a distancia, y aplicarla para llevarla cada vez más lejos en tiempo, espacio, poblaciones atendidas y profundidad de resultados. 